Medicine

Elsevier Editorial System(tm) for Preventive

Manuscript Draft

Manuscript Number: PM-16-48R1

Title: Testing behavioral interventions to optimize participation in a population-based colorectal cancer screening program in Catalonia, Spain

Article Type: Research paper

Keywords: colorectal cancer; screening; participation; uptake; behavioral interventions; reminder letter

Corresponding Author: Dr. Montse Garcia, PhD

Corresponding Author's Institution: Catalan Institute of Oncology-IDIBELL

First Author: Sandro Stoffel, BSC

Order of Authors: Sandro Stoffel, BSc; Llúcia Benito; Núria Milà; Noemie Travier; Gemma Binefa; Carmen Vidal; Jose Espinosa; Victor Moreno; Montse Garcia, PhD

Abstract: The aim of the study was to measure the effect of three behavioral interventions on participation compared to the standard invitation letter in a population-based colorectal cancer screening program in 2014. For that purpose, a four-arm randomized field trial was conducted among 5,077 individuals aged 50 to 69 years. Over a 8-week period, each week was randomly allocated to the intervention or the control conditions. Individuals assigned to the intervention conditions additionally received a prompt to write down the date to pick up the screening test in a pharmacy. Two of the three intervention groups also included an additional paragraph in the invitation letter on either: 1) the high share of individuals participating regularly (social norms condition) or 2) the importance of regular participation (benefit condition).

We measured screening participation before and after receiving a reminder letter six weeks after the screening invitation. An overall 8.0 percentage point increase in CRC screening was achieved as a direct result of receiving a reminder letter, however none of the intervention strategies influenced participation. The only significant difference was found for newly invited individuals. There, participation rates decreased from $34.9 \%$ to $24.2 \%$ when the invitation mailing mentioned the importance of regular participation (OR:0.60;95\%CI:0.38-0.95).

While none of the intervention strategies improved participation rates we found that praising the benefit of regular screening may discourage individuals who have never been invited before as the continuous behavior may be perceived as a large request. Nevertheless, the reminder letter boosted participation rates independently of the intervention assigned. 
Eduardo L. Franco, M.D., Ph.D.

Editor-in-Chief

Department of Oncology

McGill University

546 Pine Avenue Wes

Montreal, H2WaS6, Canada

Dear Dr. Franco,

I am pleased to resubmit for publication the revised version of the manuscript entitled "Testing behavioral interventions to optimize participation in a population-based colorectal cancer screening program in Catalonia, Spain. The aim of the study was to measure the effect of three behavioral interventions on screening uptake compared to the standard invitation letter. For this purpose, a four-arm randomized field trial was conducted. Over an 8-week period, each week was randomly allocated to either the intervention or the control conditions ( $n=5,077$ ), with uptake recorded before and after the reminder letter.

The manuscript was first sent to the journal on the 13rd of January 2016, and we got the answer on April 28th of the same year, where we had been told to give an answer in a short period of time (Reference: PM-16-48R1). Due to personal aspects, it's being impossible to carry out the reviewers' answers (The revision option expired). The manuscript has been changed taking into account the useful reviewers' comments and the responses have been added.

The authors of the paper directly participated in the planning, analysis, and writing of the paper, have approved the final revised version submitted, and will take public responsibility for the content of the paper.

The article is original and it is not submitted anywhere other than your journal. There is no conflict of interests regarding this investigation. We would of course be ready to provide further information about the data and methods you so desire.

Correspondence about the manuscript should be addressed to myself at the Catalan Institute of Oncology, as indicated in the title page.

Thank you very much for your kind attention. We look forward to hearing from you.

Sincerely,

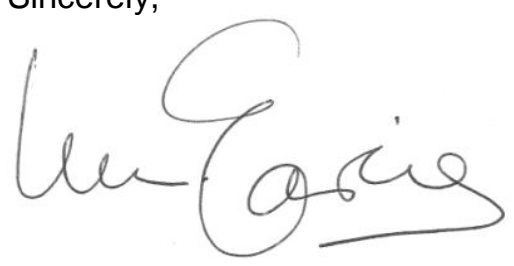

Montse Garcia, BSc, PhD

Cancer Prevention and Control Program

Catalan Institute of Oncology

Av. Gran Via 199-203

08908 Hospitalet de Llobregat (Spain) 


\section{RESPONSE POINT BY POINT TO THE REVIEWERS}

We sincerely thank the reviewers and the editor for their insightful comments. We have revised our manuscript in our effort to address all comments raised. Below you may find a point-by-point response to the reviewer's comments.

\section{Reviewer \#1:}

The manuscript reports findings from a 4-arm cluster randomized trial testing the effectiveness of different types of messaging included in mailed intervention prompting recipients to pick up and use a FIT test for colorectal cancer screening.

The authors provide theoretical support for the different types of messaging and the study design and statistical tests seem appropriate to address the research aim.

Specific concerns are outlined below.

We thank the reviewer for taking the time to read our manuscript and provide suggestions to improve the work.

1. The manuscript's introduction does not set up the problem being addressed by the intervention in a direct and succinct manner.

There are likely stronger stories about why understanding the impact of variations on the messaging used in mailed reminders should be tested.

For example, from an efficiency perspective it is important that for the same cost (i.e., paper, postage, etc.) the maximum number of tests get completed.

Thus, important to understand how to maximize the effectiveness of messaging included in letters (mailed interventions) of equal costs.

We have focused and modified the introduction to set up the problem in a direct and succinct manner.

2. Additionally, the introduction is void of any support for results being stratified by previous screening behavior.

We have added information in the introduction to explain and justify the stratification.

"The present study was designed to evaluate the effectiveness of such an intention prompt with and without an additional message that either highlights the benefit of 
regular screening or the social norm. The study describes the effects of these three cost-neutral behavioural interventions on the uptake rate before and after the standard reminder letter in a randomized field trial. Furthermore, we analyse the effectiveness of the interventions for three distinctive groups; newly invited, never screened and previous screened. These groups differ in their screening experience, attitude and knowledge of CRC screening, and have been stratified taking into account the published literature (Bulliard et al., 2014; Klabunde et al., 2015).

We hypothesized that the implementation prompt would facilitate the pick-up of the test kit and reducing the perceived barriers. Highlighting the high prevalence and importance of regular screening should mainly increase screening attendance among individuals who have refused previous screening invitations or were invited for the first time. We expect therefore the lowest effect for individuals who have participated in a previous screening round as they were already convinced of the program's purpose."

3. I found Figure 2 confusing and uninformative as currently displayed.

We have deleted Figure 2 entitled "Participation according to different interventions and stratified by previous screening behavior".

4. The last paragraph of the results section is confusing as the text indicates that there are 2 subgroups for which results were significant, but in Table 3 there is only one.

After reading the entire paragraph I understand that this is due to differences between results that account for and don't account for clustering. I'd be inclined to report only those findings that account for clustering in both the text and tables. Having said that, I worry that the significant subgroup finding is spurious.

Following the reviewer's suggestion, we report only those findings that account for clustering in both the text and tables.

We agree with the reviewer that Type I error cannot be rule out. The p-value was 0.027 ; therefore the probability of rejecting the null hypothesis given that is true was about $2.7 \%$. We have added a sentence in the discussion section.

5. Please note that first sentence of results states $34.6 \%$ participated while table 1 says $34.4 \%$.

Sorry, that was a typo. Thanks for noticing it! 
6. The conclusions are not well couched in findings from prior mailed reminder-type interventions. What types of messaging has previously been effective? Not effective? It may also be important to couch recommendations in the context of cost. If additional messaging doesn't hurt, is there a harm to including it? No reminder-type intervention has been made. The reminder was the same for all invitees, and was already used in the program before starting the study.

The study has shown the benefits of the reminder letter, but it is a strategy to improve the participation that is already implemented in our colon cancer screening program

7. Finally, there are places in the manuscript that are difficult to follow and seem challenged (e.g., uncommon or poor word choice) likely due to English not being the first language of the authors. I would recommend the authors enlist the help of a scientific writer or similar professional to assist with translation.

As suggested by the reviewer, the manuscript has been checked by an Englishspeaking colleague prior to resubmission.

\section{Reviewer \#2:}

This is a very interesting study regarding behavioural interventions to improve the attendance to CRC screening programmes. Participation rates in such programmes are still lower than the European Commission recommendations. Therefore to evaluate strategies that tend to improve the attendance is crucial.

We thank the reviewer for reading our manuscript and commenting thoughtfully on our work

1. First of all, in the Introduction:

I would suggest an alternative theoretical model to explain the factors that influence the decision of participating in such programmes.

From the social determinants of health model the "individual decision" is socially mediated (Solar O, Irwin A. A conceptual framework for action on the social determinants of health. Cairo: Commission on Social Determinants of Health; 2007) and therefore social inequalities exist, which are unjust and "avoidable".

From this point of view there are no "non-modifiable factors". Inequalities due to "demographic factors" such as age, gender, or "educational level", for example, that are classified as "non-modifiable" can be reduced with interventions specifically 
addressed to elderly people, men, or low educational level, that are those who are identified that participate to a lesser extent (Javanparast S, Ward P, Young G, Wilson C, Carter S, Misan G, et al. How equitable are colorectal cancer screening programs which include FOBTs? A review of qualitative and quantitative studies. Prev Med. 2010; 50: 165-72).

We have added some information about this theoretical model in the introduction

"Socioeconomic health differences occur when the quality of these intermediary factors are unevenly distributed between the different socioeconomic classes: socioeconomic status determines a person's behavior, life conditions, etc., and these determinants induce higher or lower prevalence of health problems. There are some social inequalities that could influence the individual decision of participation (Solar, 2007) These factors could be reduced with interventions specifically addressed to elderly people, men, or low educational level, that are those who are identified that participate to a lesser extent (Javanparast et al., 2010).

Therefore, it is important to design new behavioral interventions for promoting screening. Given the highly constrained financial resources for screening promotion, it is essential for screening organisations to seek cost-effective interventions. Previous interventions featured additional mailings, such as pre-notification and reminder letters (Power et al. 2009). While these interventions proved to be cost-effective they are not cost-neutral. Thus, before introducing additional mailings, screening organisations should try to understand how to maximize the effectiveness of the existing mailings by manipulating their presentation and content. Studies have shown the screening decision is influenced by the way information is presented (Purnell et al. 2015). The individual judgement of CRC screening depends on whether individuals understand the importance and consequence of the screening importance. Low awareness of the benefits of screening is often found among non-participants (Garcia et al. 2011). Thus, highlighting the benefit of regular screening for early detection could improve individual attitudes about CRC screening and increase participation intentions (Purnell et al. 2015)." 
2. In fact, I would substitute the term "structural barriers" for "programme organizational barriers", because the term "structural" refer to those that determine social position (education, occupation, income, gender and ethnic group), which are in turn determined by the socio-political context (governance, economic and social policy and cultural norms and values) (World Health Organization. A conceptual framework for action on the social determinants of health. Social Determinants of Health discussion paper 2. Geneva: World Health Organization; 2010).

As suggested by the reviewer, we have replaced the term "structural barriers" for "program organizational barriers".

3. On the other hand, taking into account that most of the literature shows that participation in CRC screening programme is different by sex (Javanparast $S$, Ward $P$, Young G, Wilson C, Carter S, Misan G, et al. How equitable are colorectal cancer screening programs which include FOBTs? A review of qualitative and quantitative studies. Prev Med. 2010; 50: 165-72), and that the reasons to attend differ between men and women (1.Molina-Barceló A, Salas Trejo D, Peiró-Pérez R, Málaga López A. To participate or not? Giving voice to gender and socio-economic differences in colorectal cancer screening programmes. Eur J Cancer Care (Engl). 2011; 20: 669-78. 2.Molina-Barceló A, Salas-Trejo D, Peiró-Pérez R, Vanaclocha M, Pérez E, Castán S. Reasons for participating in the Valencian Community Colorectal Cancer Screening Programme by gender, age, and social class. Rev Esp Enferm Dig. 2014 Aug;106(7):439-47) I would suggest to include the analysis of the results by sex. Maybe the Results could be better understood.

We computed stratified models by sex, since men and women have shown differences in screening participation rates. However, after we checked there were no relevant differences according to sex and in order not to reduce the power of the study, we decided to present the results from the models adjusted for sex and age. 
Table 1.

Males

\begin{tabular}{|c|c|c|c|c|c|c|}
\hline \multirow[t]{2}{*}{$(n=2,423)$} & \multicolumn{3}{|c|}{$\begin{array}{l}\text { Initial participation } \\
\text { (before reminder) }\end{array}$} & \multicolumn{3}{|c|}{$\begin{array}{l}\text { Final participation } \\
\text { (after reminder) }\end{array}$} \\
\hline & \multirow{2}{*}{$\begin{array}{l}\% \\
6.2\end{array}$} & \multicolumn{2}{|c|}{$95 \% \mathrm{Cl}$} & \multirow{2}{*}{$\begin{array}{l}\% \\
9.9\end{array}$} & \multicolumn{2}{|c|}{$95 \% \mathrm{Cl}$} \\
\hline Never Screened & & $5.1-$ & 7.6 & & 8.4- & 11.5 \\
\hline Newly invited & 15.0 & 11.7- & 19.0 & 28.1 & $23.7-$ & 32.9 \\
\hline \multicolumn{7}{|l|}{ Previously } \\
\hline screened & 65.7 & 61.9 & 69.2 & 78.8 & $75.5-$ & 81.8 \\
\hline Overall & 23.2 & 21.5 & 24.9 & 29.3 & 27.6- & 31.0 \\
\hline
\end{tabular}

Females

\begin{tabular}{|c|c|c|c|c|c|}
\hline \multirow[t]{2}{*}{$(n=2,654)$} & \multicolumn{2}{|c|}{$\begin{array}{l}\text { Initial participation } \\
\text { (before reminder) }\end{array}$} & \multicolumn{3}{|c|}{$\begin{array}{l}\text { Final participation } \\
\text { (after reminder) }\end{array}$} \\
\hline & $\%$ & $95 \% \mathrm{Cl}$ & $\%$ & $95 \% \mathrm{Cl}$ & \\
\hline Never screened & 8 & $6.7-9.5$ & 12.9 & 11.3- & 14.8 \\
\hline Newly invited & 22.1 & $18.2-26.6$ & 33.4 & 28.8- & 38.4 \\
\hline \multicolumn{6}{|l|}{ Previously } \\
\hline screened & 68.6 & 65.4- 71.7 & 81.9 & $79.2-$ & 84.3 \\
\hline Overall & 29.3 & $27.6-\quad 31.0$ & 37.8 & $35.9-$ & 39.6 \\
\hline
\end{tabular}


Table 3.

Males

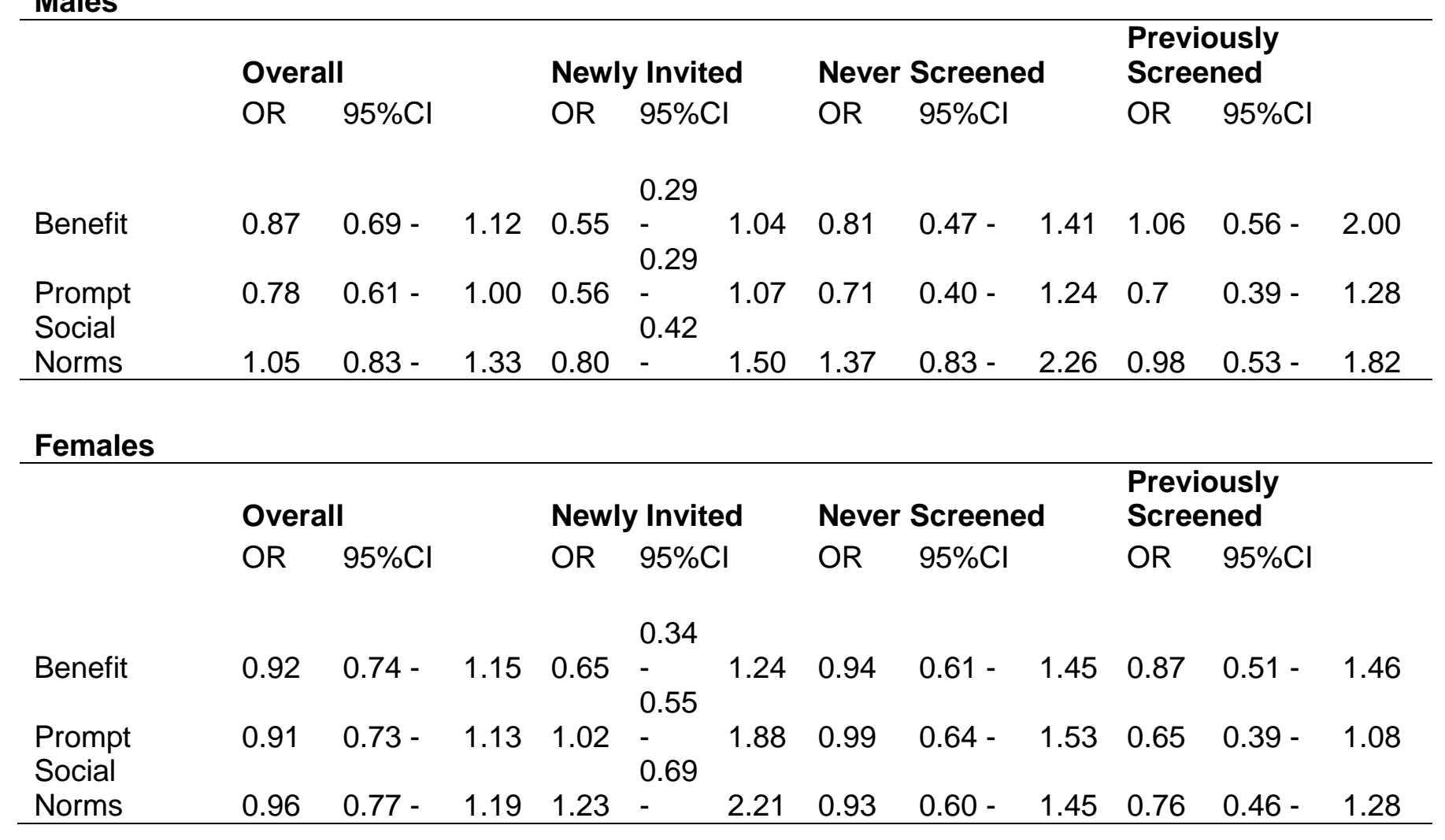


4. Finally, in my opinion the two messages would it be better to measure neither adherence nor attendance, because both highlight the continuity in the programme ("... continue in the programme..." and "Regular participation...". Maybe this could be one of the reasons why the "regular participation benefit" discourages first participants. This could be included in the Discussion.

The reviewer has raised a good point. We agree with him/her that the two text messages do not directly deal with attendance as they focus on continuity. Thus the messages should not increase participation of newly invited but rather discourage them. We have added a sentence in the Discussion section.

\section{Minor corrections:}

5. Introduction, Line 2: include the first abbreviation of CRC in order to use it along the paper Invitation mailing conditions, Line 54: an end quotation mark is needed; Line 56: letter than latter Study population, Line 2: delete the point after parenthesis; Line 6: replace the comma by a point

The requested changes have been made.

\section{Reviewer \#3:}

No comments provided that could be shared with authors.

\section{EDITORIAL OFFICE'S ADDITIONAL REVISION INSTRUCTIONS:}

1. Please provide a completed CONSORT checklist.

As suggested by the reviewer, we have added the completed CONSORT checklist.

2. You may use up to 3500 words for your revised manuscript (main text) (and a maximum of 250 words for the abstract), including the additional text required to address the points raised in the critiques.

The text and the summary have been both adjusted to the journal requirements.

3. We ask you to include word counts for the abstract and for the main text on the cover page. Please take into account also the editorial instructions in the body of this email. We have added this information on the cover page. 
4. Although we expect that you will address the comments by the reviewers in your response letter we also would like to see the corresponding revision in the text of the manuscript. Some of the reviewers' comments may seem to be simple queries or challenges that do not prompt revisions to the text. Please keep in mind, however, that readers may have the same perspective as the reviewer. Therefore, it is essential that you attempt to amend or expand the text to clarify the narrative accordingly.

We have adapted the text of the manuscript taking into account all the reviewers' comments. The modified text has been written in red.

5. Previous research supports the importance of provider recommendations for CRC screening, and patients who have never completed CRC screening may need provider counseling before completing a FIT.

A systematic review study showed that an advance notification letter, postal mailing, written reminders increase uptake of faecal tests for CRC screening uptake and could be easily implemented (Rat el al., 2018). Some studies assessed the effect of different frames of invitation messages (varying presentation and content of the written information). They showed increases in uptake, on the basis of a leaflet containing information on FOBT or an 'implementation intention' technique (Rat et al., 2018).

Previous research supports that the low prevalence of screening for colorectal cancer appears to be due to lack of awareness and inadequate provider counseling rather than poor patient acceptance for screening. Systematic counseling about colorectal cancer screening will likely improve screening rates and reduce disparities by race/ethnicity and education (Gilbert and Kanarek, 2005; Wee, McCarthy and Phillips, 2005). Nevertheless, communication interventions to improve CRC screening rates must therefore be appropriate in terms of cognitive and health literacy demands (Smith et al., 2013). Feedback between the CRC screening programme and primary health care professionals is essential to ensure the quality of the screening programme and consistent monitoring criteria (Benito et al., 2016). 


\section{Highlights}

- Behavioral interventions were designed to promote colorectal cancer screening.

- The reminder letter increased screening uptake significantly.

- None of the intervention strategies improved overall participation.

- Praising the benefit of regular screening discouraged newly invited individuals.

- Proof-of-concept experiments are paramount to generate reliable evidence. 
Testing behavioral interventions to optimize participation in a population-based colorectal cancer screening program in Catalonia, Spain

S. Stoffel ${ }^{1}$, L. Benito ${ }^{2}$, N. Milà ${ }^{3,4}$, N. Travier ${ }^{3}$, G. Binefa ${ }^{3}$, C. Vidal, J. Espinosa ${ }^{3}$, V. Moreno ${ }^{3}$, M. Garcia $^{3}$

1. Research Department of Behavioural Science and Health, University College London, London, UK

2. Department of Fundamental Care and Medical-Surgical Nursing, University of Barcelona, L'Hospitalet de Llobregat, 08907 Barcelona, Spain

3. Cancer Prevention and Control Program, Catalan Institute of Oncology, IDIBELL, L'Hospitalet de Llobregat, 08908 Barcelona, Spain

4. Consortium for Biomedical Research in Epidemiology and Public Health (CIBEResp), 28029, Madrid, Spain

\section{Address correspondence to:}

Montse Garcia, Cancer Prevention and Control Unit; Catalan Institute of Oncology; Av. Gran Via 199-203; 08908 L'Hospitalet de Llobregat. Telf: +34 932607186 - Fax: +34 932607956

E-mail: mgarcia@iconcologia.net 


\section{Abstract}

The aim of the study was to measure the effect of three cost-neutral behavioral interventions on participation compared to the standard invitation letter in a population-based colorectal cancer screening program in 2014. For that purpose, a four-arm randomized field trial was conducted among 5,077 individuals aged 50 to 69 years. Over an 8-week period, each week was randomly allocated to the intervention or the control conditions. Individuals assigned to the intervention conditions additionally received a prompt to write down the date to pick up the screening test in a pharmacy. Two of the three intervention groups also included an additional paragraph in the invitation letter on either: 1) the high proportion of individuals participating regularly (social norms condition) or 2) the importance of regular participation (benefit condition).

We measured screening participation before and after receiving a reminder letter six weeks after the screening invitation. An overall 8.0 percentage point increase in CRC screening was achieved as a direct result of receiving a reminder letter; however none of the intervention strategies influenced participation. The only significant difference was found for newly invited individuals. There, participation rates decreased from $34.9 \%$ to $24.2 \%$ when the invitation mailing mentioned the importance of regular participation (OR: $0.60 ; 95 \% \mathrm{Cl}: 0.38-0.95$ ).

While none of the intervention strategies improved participation rates we found that praising the benefit of regular screening may discourage individuals who have never been invited before as the continuous behaviour may be perceived as a large request. Nevertheless, the reminder letter boosted participation rates independently of the intervention assigned.

Key words: colorectal cancer screening; participation; uptake; behavioural interventions; reminder letter 


\section{Introduction}

Reductions in mortality seen in randomized trials for colorectal cancer (CRC) screening can only be reproduced in the wider population if uptake is adequate. (Weller et al. 2009). Low participation rates would dilute the intrinsic efficacy of CRC screening test, reducing the overall yield for advanced neoplasia in a population setting (Hassan et al. 2012).

Although participation in CRC screening varies widely between regions and countries, screening programs generally remain underutilized and participant numbers typically compare poorly with those for breast and cervical cancer screening (Klabunde et al. 2015). Participation rates in countries with organized screening programmes based on faecal tests are low, ranging from $22.80 \%$ to $62.80 \%$ and with disparities across socioeconomic groups (Karsa et al., 2008). There are some social inequalities that could influence the individual decision of participation (Solar, 2007). Some studies shows that people with lower socio-economic status (SES) have less knowledge about the screening programme and the disease and often have difficulty understanding written information, which hinders participation. (Molina-Barcelo et al., 2011). The inequalities could be reduced with interventions specifically addressed to elderly people, men, or low educational level, that are those who are identified that participate to a lesser extent (Baker et al., 2014; Javanparast et al., 2010)

Therefore, it is important to design new behavioral interventions for promoting screening. Given the highly constrained financial resources for screening promotion, it is essential for screening organisations to seek cost-effective interventions. Previous interventions featured additional mailings, such as pre-notification and reminder letters (Power et al. 2009). While these interventions proved to be cost-effective they are not cost-neutral. Thus, before introducing additional mailings, screening organisations should try to understand how to maximize the effectiveness of the existing mailings by manipulating their presentation and content. Studies have shown the screening decision is influenced by the way information is presented (Purnell et al. 2015). The individual judgement of CRC screening depends on whether individuals understand the importance and consequence of the screening importance. Low awareness of the benefits of screening is often found among non-participants (Garcia et al. 2011). Thus, highlighting the benefit of regular screening for early detection could improve individual attitudes about CRC screening and increase participation intentions (Purnell et al. 2015). 
Similarly, including a message in the invitation letter that a large majority of individuals participate regularly in the screening program should increase participation rates. A recent study on men found that communicating high participation rates in CRC screening programmes increases intentions to be screened (Senore et al., 2015). The rationale for this social norm effect is based on two consistent findings: (i) Individuals may underestimate the prevalence of screening participation, and (ii) individuals use the perceptions of their peer's behaviour as a standard against which to compare their own intentions (Cialdini et al. 1990).

Although, individuals may judge the screening program useful, they may still fail to participate as they face programme organizational barriers. Possible interventions aimed at reducing such barriers focus mainly on improving access by reducing the required participation effort (Brouwers et al. 2011). Possible examples are mailing out test kits to eligible individuals and offering additional information about the screening program. While sending out test kits with the invitation letter reduces the required effort for participation it is not cost-efficient as not all individuals have intentions to get screened. Up to date, the Catalan CRC screening program uses therefore an alternative procedure in which eligible individuals can pick up a free FIT kit from a pharmacy engaged to the program. This procedure may be cost-effective but present a potential barrier for participants as they have to get the test kit themselves. Individuals may be interested to participate in the screening program but unwilling to go to the pharmacy or procrastinate the pick-up. A recent study demonstrated that prompting individuals to develop a plan for when to perform the desired behaviour is enough to increase immunization behaviour (Milkman et al. 2011). Such prompts to form an implementation intention has been shown to be effective in promoting the initiation of goal striving for individuals who have problems with action control or over-extend their capability to regulate their behavior (Gollwitzer 1999; Gollwitzer and Sheeran 2006). If the desired behavior consists of a time-consuming goal, such as picking up a FIT kit from a pharmacy, then simply forming the respective goal intention could facilitate its achievement. The prompt to plan the pick-up by writing down the desired date on the list of the eligible pharmacies can help individuals to achieve it. Similarly, sending out a reminder letter helps individuals remembering the request (Senore et al. 2015).

Furthermore, in the present study we analyse the effectiveness of the interventions for three distinctive groups; newly invited, never screened and previous screened. These groups differ in 
their screening experience, attitude and knowledge of CRC screening, and have been stratified taking into account the published literature (Bulliard et al., 2014; Klabunde et al., 2015).

The present study was designed to evaluate the effectiveness of such an intention prompt with and without an additional message that either highlights the benefit of regular screening or the social norm. The study describes the effects of these three cost-neutral behavioural interventions on the uptake rate before and after the standard reminder letter in a randomized field trial.

We hypothesized that the implementation prompt would facilitate the pick-up of the test kit and reducing the perceived barriers. Highlighting the high prevalence and importance of regular screening should mainly increase screening attendance among individuals who have refused previous screening invitations or were invited for the first time. We expect therefore the lowest effect for individuals who have participated in a previous screening round as they were already convinced of the program's purpose.

\section{Methods}

\section{Screening Procedure}

In 2000, a biennial fecal occult blood test-based CRC screening program was launched in L'Hospitalet of Llobregat, an industrial city in the metropolitan area of Barcelona (Catalonia, Spain). The target population includes all men and women aged 50 - 69 years who lived in the city (average of 65,000). From 2010, a quantitative Fecal Immunochemical Test (FIT) has been used as a primary screening tool (OC-Sensor, Eiken Chemical Co., Japan). Up to date, The FIT is distributed and collected through community pharmacies.

Demographic data on this population was gathered from the Primary Healthcare Information System. L'Hospitalet is divided into 12 basic healthcare areas and each individual is uniquely allocated to a general practitioner in one of these areas. Screening invitations were sent personally and sequentially to eligible population assigned to each one of the Basic Healthcare Areas.

Subjects who did not meet the inclusion criteria for CRC screening were excluded according to the following criteria: personal history of CRC or adenomas, familial CRC cancer, inflammatory bowel disease, colonoscopy in the previous 5 years or faecal occult blood test (FOBT) in the 
previous 2 years, terminal disease or severe disabling condition. Subjects with an invalid mailing address and removals from the screening area registry were also excluded because they could not be invited to screening.

Eligible subjects were invited by a personal letter offering a test completely free, a leaflet with information regarding $\mathrm{CRC}$ and the screening process and a list of the nearest pharmacies participating in the program. A reminder letter was sent out if no response was obtained after six weeks of the initial invitation.

A detailed description of the FIT performance is provided elsewhere (Garcia et al. 2015). Briefly, participants collected one sample of feces from one bowel movement and a $100 \mathrm{ng} \mathrm{Hb} / \mathrm{ml}$ cutoff (20 mg Hb/g feces) was used as the threshold for test positivity. Those individuals with a positive test were offered a colonoscopy for diagnostic confirmation.

\section{$\underline{\text { Randomized field trial }}$}

We conducted a four-arm randomized field trial to investigate the efficacy of the implementation of an intention prompt and the additional messages.

\section{Invitation mailing conditions}

As behavioral interventions are most likely to influence screening if they target more than one factor, we combined the implementation prompt in two experimental conditions with either the message about the high prevalence or importance of regular screening (Power et al. 2009; Camiloni et al. 2013; Senore et al. 2015).

The control group was the standard invitation letter used in the other screening areas In addition, the three intervention conditions prompted recipients to write down the date they planned to pick up their test kit and two of them included additional information about injunctive social norms or benefit of early detection. A standard reminder letter was sent out if no response was obtained after six weeks of the initial invitation.

The intention prompt on top of the sheet with the engaged pharmacies was adapted from a previous study and contained the sentence: "The majority people find it helpful to write down the date they want to pick up their test kit" (Milkman et al. 2011). The normative message on the social norms condition stated the proportion of individuals who engage in the desired behavior (Jemmot et al. 1986). The sentence said: "The majority of people participating for the first time continue in the program later on. Follow their example and participate now." The benefit 
condition added the following sentence to the invitation letters: "Regular participation in the screening program increases the chance of early detection and facilitates treatment." The reminder letter was sent out six weeks after the initiation letter and was identical for all experimental conditions.

\section{Study population}

Our study population was 5,077 inhabitants (from a basic healthcare area) eligible for the sixth biennial CRC screening program of L'Hospitalet of Llobregat. These subjects were invited from the $12^{\text {th }}$ of May to the $4^{\text {th }}$ of July 2014 ,

A quarter of the population was assigned to each experimental condition $(n=4)$. Intervention and control groups were randomized along the 8 week-period to prevent summer break effects. Figure 1 shows the component of the mailer that varied across the four conditions and the distribution of invitations throughout the period of study. There were no differences in the distribution of individuals between the four mailing conditions in terms of sex $(p=0.78)$, age $(p=0.64)$ and CRC screening experience $(p=1.00)$.

The study protocol was approved by the Clinical Research Ethics Committee of the Bellvitge University Hospital, and all involved parties followed the ethical requirements set forth in the Spanish Organic Law on Protection of Personal Data (15/1999 of December 13).

The outcomes of interest were participation rates according to each experimental condition before and after the reminder letter.

We performed a logistic regression with random intercept analysis to compare each mailing condition versus the standard invitation letter, adjusting for sex and age group. We included the invitation week as random effects intercept to account for the cluster variability. We stratified the model by previous screening behavior. We reported the odds ratio (OR) adjusted for age and sex and $95 \%$ confidence intervals $(95 \% \mathrm{Cl})$. This study had $80 \%$ power to detect differences in participation of $5.5 \%$ between control and experimental groups.

\section{Results}


The overall participation in the CRC screening program was $34.6 \%$. From those individuals who picked-up the FIT at the pharmacies, $96.8 \%$ completed and returned it (data not shown).

Table 1 shows the effect of reminder letter on participation rates according to previous screening behavior. An overall 8.0 percentage point increase in CRC screening was achieved, which translates to an additional 409 individuals who were screened, as a direct result of receiving a reminder. Differential rates were statistically significant in all groups (newly invited, never screened and previous screened).

Table 2 presents participation rates according to the different behavioral interventions. Overall, participation rates among those individuals allocated in the prompt and the benefit conditions were lower than those in the control and norms condition.

Multivariate logistic regression analysis showed that the odds for completing a screening test were significantly lower for two subgroups (table 3). Newly invited individuals were less likely to hand in their stool samples if they were in the benefit condition (OR: 0.60; 95\% Cl: 0.38-0.95). On the other hand, individuals who had been previously screened might be less likely to participate when they were allocated in the prompt condition compared to those who got the standard invitation letter. Although after adjusting for the cluster effect the differences were not statistically significant (OR: 0.68; 95\% Cl: 0.40-1.17).

\section{Discussion}

While none of our three experimental conditions increased screening behavior, we found that highlighting the benefit of continuous behavior may discourage individuals to adapt. The results of this study demonstrate a clear demotivating effect of praising the benefits of regular participation on individuals who had never been invited before. In designing invitation mailings, one should be very cautious in stating the desired behavior, because repeated behavior may be perceived as a large request. Studies have shown that individuals are more likely to agree to a small than a large request (Freedman and Fraser, 1996; Dolin and Booth-Butterfield, 1995). Essentially, the more an individual goes along with small requests, like participating in a screening program one time, the more likely is it that the individual participates also in future programs. In this sense, the newly invited individual may have felt obligated to participate regularly. 
The insignificant impact of the implementation prompt suggests that individuals do not have problem to plan the pick-up of their test kit. Note, that this result does not contradict the positive finding of the implementation prompt in the context of vaccination. It may be that getting a flu shot is more time consuming and therefore more difficult to plan than passing by a pharmacy to pick up the test kit. This argument is supported by the finding of the original study that the implementation prompt had a larger impact at one-day clinic sites than at multi-day clinic sites (Milkman et al, 2011). The authors believe that the implementation intentions prompts are most effective when the opportunity for participation is fleeting. In our case, the pharmacies are at least as easily accessible as multi-day clinics and the implementation prompt may therefore not be needed.

Differently, the insignificant effect of the social norms treatment may have been caused by the vague description of the participation rate. Stating that the majority of individuals participating for the first time continue in the program later on does not give individuals a clear picture of the social norm. It may be that individuals think that only slightly more than $50 \%$ of the individuals continue with the program. A future study should therefore investigate whether communicating the observed $80 \%$ participation rate of individuals who have participated previously affects screening behavior.

Finally, we found that the neutral reminder letter increased screening rates, independently from the individual's experience with the screening program $(p<0.01)$. This positive effect of the reminder letter is persistent with previous research (Senore et al. 2015; Brouwers et al. 2011a; Brouwers et al. 2011b; Sabatino et al. 2012).

A systematic review study showed that an advance notification letter, postal mailing, written reminders increase uptake of faecal tests for CRC screening uptake and could be easily implemented (Rat el al., 2018). Some studies assessed the effect of different frames of invitation messages (varying presentation and content of the written information). They showed increases in uptake, on the basis of a leaflet containing information on FOBT or an 'implementation intention' technique (Rat et al., 2018).

Previous research supports that the low prevalence of screening for colorectal cancer appears to be due to lack of awareness and inadequate provider counseling rather than poor patient acceptance for screening. Systematic counseling about colorectal cancer screening will likely 
improve screening rates and reduce disparities by race/ethnicity and education (Gilbert and Kanarek, 2005; Wee, McCarthy and Phillips, 2005). Nevertheless, communication interventions to improve CRC screening rates must therefore be appropriate in terms of cognitive and health literacy demands (Smith et al., 2015). Feedback between the CRC screening programme and primary health care professionals is essential to ensure the quality of the screening programme and consistent monitoring criteria (Benito et al., 2016)

This study has some limitations, we could not randomize at individual level because of the CRC screening invitation process. CRC screening territory is divided according to healthcare basic areas, and individuals are invited sequentially. As cluster randomization is more prone to bias than individual randomization we included the invitation week as a random effect in the model to account for that variability.

Although the impacts of our experimental conditions were mostly insignificant, our study shows that importance of proof-of-concept experiments to generate reliable evidence of the interventions. Implementation prompts have been used successfully in the context of vaccination (Milkman et al. 2011), but they did not work in a setting where participation was less time consuming. It is therefore important to provide reliable evidence of the impact of behavioral interventions for each application. A basic requirement for scientific integrity is the ability to replicate the results of research and apply them to different settings. These proof-of-concept studies are particularly important to generalize findings.

\section{Acknowledgments}

This study was partially co-funded by the Carlos III Health Institute and the European Regional Development Fund - a way to build Europe (PI05/0942, RD12/003670053), and by the Department of Universities and Research (2014SGR635, 2014SGR647), Government of Catalonia.

\section{Declaration of conflicting interests}

The authors declared no potential conflict of interest with respect to the research, authorship and /or publication of this article. 


\section{References}

Baker, D. W., Brown, T., Buchanan, et al. (2014). Comparative effectiveness of a multifaceted intervention to improve adherence to annual colorectal cancer screening in community health centers: a randomized clinical trial. JAMA internal medicine, 174(8), 1235-1241.

Benito, L., García, M., Binefa, G., et al. (2016). Cross-sectional survey on awareness of colorectal cancer and a screening programme for primary health care professionals in Catalonia, Spain. European journal of cancer care, 25(6), 992-1004.

Brouwers MC, De Vito C, Bahirathan L, et al (2011) Effective interventions to facilitate the uptake of breast, cervical and colorectal cancer screening: an implementation guideline. Implement Sci;6(1):112.

Brouwers MC, De Vito C, Bahirathan L, et al (2011) What implementation interventions increase cancer screening rates? a systematic review. Implement Science; 6 (1): 111.

Bulliard JL, Garcia M, Blom J, Senore C, Mai V, Klabunde C. Sorting out measures and definitions of screening participation to improve comparability: The example of colorectal cancer. Eur J Cancer 2014;50(2): 434-46.

Camilloni L, Ferroni E, Cendales BJ, et al (2013) Methods to increase participation in organized screening programs: a systematic review. BMC Public Health;13 (1): 464.

Cialdini RB, Reno RR, Kallgren CA (1990) A focus theory of normative conduct: recycling the concept of norms to reduce littering in public places. J Pers Soc Psychol; 58(6):1015-26.

Dolin DJ, Booth-Butterfield S (1995) Foot-in-the-door and cancer prevention. Health Commun;7(1):55-66.

Freedman JL, Fraser SC (1996) Compliance without pressure: the foot-in-the-door technique. J Pers Soc Psychol;4(2):195-202.

Garcia M, Borràs JM, Milà N, et al (2011) Factors associated with initial participation in a population-based screening for colorectal cancer in Catalonia, Spain: a mixed-methods study. Prev Med;52 (3):265-7

Garcia M, Milà N, Binefa G et al (2015) Fecal hemoglobin concentration as a measure of risk to tailor colorectal cancer screening: are we there yet? Eur J Cancer Prev;24(4):321-27.

Gilbert A, Kanarek N. Colorectal cancer screening: physician recommendation is influential advice to Marylanders. Prev Med. 2005;41(2):367-79. doi:10.1016/j.ypmed.2005.01.008.

Gollwitzer PM (1999) Implementation intentions: strong effects of simple plans. Am Psychol; 54 (7): 493-503.

Gollwitzer PM, Sheeran P (2006) Implementation intentions and goal achievement: A meta-analysis of effects and processes. Adv Exp Soc Psychol; 38: 69-119.

Hassan C, Giorgi Rossi P, Camilloni L, et al (2012) Meta-analysis: adherence to colorectal cancer screening and the detection rate for advanced neoplasia, according to the type of screening test. Aliment Pharmacol Ther; 36 (10):929-40.

Javanparast, S., Ward, P., Young, G et al. (2010). How equitable are colorectal cancer screening programs which include FOBTs? A review of qualitative and quantitative studies. Preventive medicine, 50(4), 165-172. 
Jemmott JB , Ditto PH, Coryle RT (1986). Judging health status: effects of perceived prevalence and personal relevance. J Pers Soc Psychol;50(5):899-905.

Karsa, L. V., Anttila, A., Ronco, G., Ponti, A., Malila, N., Arbyn, M., ... \& Hery, C. (2008). Cancer screening in the European Union. Report on the implementation of the Council Recommendation on cancer screening. Cancer screening in the European Union. Report on the implementation of the Council Recommendation on cancer screening.

Klabunde C, Blom J, Bulliard JL, et al. (2015) Participation rates for organized colorectal cancer screening programmes: an international comparison. J Med Screen; 22 (3):119-26.

Milkman KL, Beshears J, Choi JJ, et al (2011) Using implementation intentions prompts to enhance influenza vaccination rates. Proc Natl Acad Sci USA.; 108 (26): 10415-20.

Molina-Barceló, A., Salas Trejo, D., Peiró-Pérez, R., \& Málaga López, A. (2011). To participate or not? Giving voice to gender and socio-economic differences in colorectal cancer screening programmes. European journal of cancer care, 20(5), 669-678.

Power E, Miles A, von Wagner C, et al (2009) Uptake of colorectal cancer screening: system, provider and individual factors and strategies to improve participation. Future Oncol; 5(9):137188.

Purnell JQ, Thompson T, Kreuter MW, McBride TD. Behavioral Economics: "Nudging" Underserved Populations to Be Screened for Cancer. Prev Chronic Dis 2015;12:140346. DOI: http://dx.doi.org/10.5888/ pcd12.140346

Rat, C., Latour, C., Rousseau, R., Gaultier, A., Pogu, C., Edwards, A., \& Nguyen, J. M. (2018). Interventions to increase uptake of faecal tests for colorectal cancer screening: a systematic review. European Journal of Cancer Prevention, 27(3), 227-236.

Sabatino SA, Lawrence B, Elder R, et al (2012). Community Preventive Services Task Force. Effectiveness of interventions to increase screening for breast, cervical, and colorectal cancers: nine updated systematic reviews for the guide to community preventive services. Am J Prev Med;43(1):97-118.

Senore C, Inadomi J, Segnan N, Bellisario C, et al (2015) Optimising colorectal cancer screening acceptance: a review. Gut; 64 (7):1158-77.

Smith SG, Vart G, Wolf MS, Obichere A, Baker HJ, Raine R, Wardle J, von Wagner C. How do people interpret information about colorectal cancer screening: observations from a think-aloud study. Health Expect. 2015 Oct;18(5):703-14. doi: 10.1111/hex.12117.

Solar O, Irwin A. A conceptual framework for action on the social determinants of health. Social Determinants of Health Discussion. Paper 2 (Policy and Practice).

Wee CC, McCarthy EP, Phillips RS. Factors associated with colon cancer screening: the role of patient factors and physician counseling. Prev Med. 2005;41(1):23-9.

Weller DP, Patnick J , Mclntosh HM, et al (2009) Uptake in cancer screening programmes. The Lancet Oncol; 10 (7):693-9. 
Figure 1: Component of the invitation mailers 


\section{Testing behavioral interventions to optimize participation in a population-based colorectal cancer screening program in Catalonia, Spain}

S. Stoffel ${ }^{1}$, L. Benito ${ }^{2}$, N. Mila ${ }^{3,4}$, N. Travier ${ }^{3}$, G. Binefa $^{3}$, C. Vidal, J. Espinosa ${ }^{3}$, V. Moreno ${ }^{3}$, M. Garcia $^{3}$

1. Research Department of Behavioural Science and Health, University College London, London, UK

2. Department of Fundamental Care and Medical-Surgical Nursing, University of Barcelona, L'Hospitalet de Llobregat, 08907 Barcelona, Spain

3. Cancer Prevention and Control Program, Catalan Institute of Oncology, IDIBELL, L'Hospitalet de Llobregat, 08908 Barcelona, Spain

4. Consortium for Biomedical Research in Epidemiology and Public Health (CIBEResp), 28029, Madrid, Spain

\section{Address correspondence to:}

Montse Garcia, Cancer Prevention and Control Unit; Catalan Institute of Oncology; Av. Gran Via 199-203; 08908 L'Hospitalet de Llobregat. Telf: +34 932607186 - Fax: +34 932607956

E-mail: $\underline{\text { mgarcia@iconcologia.net }}$ 


\section{Abstract}

The aim of the study was to measure the effect of three cost-neutral behavioral interventions on participation compared to the standard invitation letter in a population-based colorectal cancer screening program in 2014. For that purpose, a four-arm randomized field trial was conducted among 5,077 individuals aged 50 to 69 years. Over an 8-week period, each week was randomly allocated to the intervention or the control conditions. Individuals assigned to the intervention conditions additionally received a prompt to write down the date to pick up the screening test in a pharmacy. Two of the three intervention groups also included an additional paragraph in the invitation letter on either: 1) the high proportion of individuals participating regularly (social norms condition) or 2) the importance of regular participation (benefit condition).

We measured screening participation before and after receiving a reminder letter six weeks after the screening invitation. An overall 8.0 percentage point increase in CRC screening was achieved as a direct result of receiving a reminder letter; however none of the intervention strategies influenced participation. The only significant difference was found for newly invited individuals. There, participation rates decreased from $34.9 \%$ to $24.2 \%$ when the invitation mailing mentioned the importance of regular participation (OR: $0.60 ; 95 \% \mathrm{Cl}: 0.38-0.95$ ).

While none of the intervention strategies improved participation rates we found that praising the benefit of regular screening may discourage individuals who have never been invited before as the continuous behaviour may be perceived as a large request. Nevertheless, the reminder letter boosted participation rates independently of the intervention assigned.

Key words: colorectal cancer screening; participation; uptake; behavioural interventions; reminder letter 


\section{Introduction}

Reductions in mortality seen in randomized trials for colorectal cancer (CRC) screening can only be reproduced in the wider population if uptake is adequate. (Weller et al. 2009). Low participation rates would dilute the intrinsic efficacy of CRC screening test, reducing the overall yield for advanced neoplasia in a population setting (Hassan et al. 2012).

Although participation in CRC screening varies widely between regions and countries, screening programs generally remain underutilized and participant numbers typically compare poorly with those for breast and cervical cancer screening (Klabunde et al. 2015). Participation rates in countries with organized screening programmes based on faecal tests are low, ranging from $22.80 \%$ to $62.80 \%$ and with disparities across socioeconomic groups (Karsa et al., 2008). There are some social inequalities that could influence the individual decision of participation (Solar, 2007). Some studies shows that people with lower socio-economic status (SES) have less knowledge about the screening programme and the disease and often have difficulty understanding written information, which hinders participation. (Molina-Barcelo et al., 2011). The inequalities could be reduced with interventions specifically addressed to elderly people, men, or low educational level, that are those who are identified that participate to a lesser extent (Baker et al., 2014; Javanparast et al., 2010)

Therefore, it is important to design new behavioral interventions for promoting screening. Given the highly constrained financial resources for screening promotion, it is essential for screening organisations to seek cost-effective interventions. Previous interventions featured additional mailings, such as pre-notification and reminder letters (Power et al. 2009). While these interventions proved to be cost-effective they are not cost-neutral. Thus, before introducing additional mailings, screening organisations should try to understand how to maximize the effectiveness of the existing mailings by manipulating their presentation and content. Studies have shown the screening decision is influenced by the way information is presented (Purnell et al. 2015). The individual judgement of CRC screening depends on whether individuals understand the importance and consequence of the screening importance. Low awareness of the benefits of screening is often found among non-participants (Garcia et al. 2011). Thus, highlighting the benefit of regular screening for early detection could improve individual attitudes about CRC screening and increase participation intentions (Purnell et al. 2015). 
Similarly, including a message in the invitation letter that a large majority of individuals on men found that communicating high participation rates in CRC screening programmes increases intentions to be screened (Senore et al., 2015). The rationale for this social norm effect is based on two consistent findings: (i) Individuals may underestimate the prevalence of screening participation, and (ii) individuals use the perceptions of their peer's behaviour as a standard against which to compare their own intentions (Cialdini et al. 1990).

Although, individuals may judge the screening program useful, they may still fail to participate as they face programme organizational barriers. Possible interventions aimed at reducing such barriers focus mainly on improving access by reducing the required participation effort (Brouwers et al. 2011). Possible examples are mailing out test kits to eligible individuals and offering additional information about the screening program. While sending out test kits with the invitation letter reduces the required effort for participation it is not cost-efficient as not all individuals have intentions to get screened. Up to date, the Catalan CRC screening program uses therefore an alternative procedure in which eligible individuals can pick up a free FIT kit from a pharmacy engaged to the program. This procedure may be cost-effective but present a potential barrier for participants as they have to get the test kit themselves. Individuals may be interested to participate in the screening program but unwilling to go to the pharmacy or procrastinate the pick-up. A recent study demonstrated that prompting individuals to develop a plan for when to perform the desired behaviour is enough to increase immunization behaviour (Milkman et al. 2011). Such prompts to form an implementation intention has been shown to be effective in promoting the initiation of goal striving for individuals who have problems with action control or over-extend their capability to regulate their behavior (Gollwitzer 1999; Gollwitzer and Sheeran 2006). If the desired behavior consists of a time-consuming goal, such as picking up a FIT kit from a pharmacy, then simply forming the respective goal intention could facilitate its achievement. The prompt to plan the pick-up by writing down the desired date on the list of the eligible pharmacies can help individuals to achieve it. Similarly, sending out a reminder letter helps individuals remembering the request (Senore et al. 2015).

Furthermore, in the present study we analyse the effectiveness of the interventions for three distinctive groups; newly invited, never screened and previous screened. These groups differ in 
their screening experience, attitude and knowledge of CRC screening, and have been stratified taking into account the published literature (Bulliard et al., 2014; Klabunde et al., 2015).

The present study was designed to evaluate the effectiveness of such an intention prompt with and without an additional message that either highlights the benefit of regular screening or the social norm. The study describes the effects of these three cost-neutral behavioural interventions on the uptake rate before and after the standard reminder letter in a randomized field trial.

We hypothesized that the implementation prompt would facilitate the pick-up of the test kit and reducing the perceived barriers. Highlighting the high prevalence and importance of regular screening should mainly increase screening attendance among individuals who have refused previous screening invitations or were invited for the first time. We expect therefore the lowest effect for individuals who have participated in a previous screening round as they were already convinced of the program's purpose.

\section{Methods}

\section{Screening Procedure}

In 2000, a biennial fecal occult blood test-based CRC screening program was launched in L'Hospitalet of Llobregat, an industrial city in the metropolitan area of Barcelona (Catalonia, Spain). The target population includes all men and women aged $50-69$ years who lived in the city (average of 65,000). From 2010, a quantitative Fecal Immunochemical Test (FIT) has been used as a primary screening tool (OC-Sensor, Eiken Chemical Co., Japan). Up to date, The FIT is distributed and collected through community pharmacies.

Demographic data on this population was gathered from the Primary Healthcare Information System. L'Hospitalet is divided into 12 basic healthcare areas and each individual is uniquely allocated to a general practitioner in one of these areas. Screening invitations were sent personally and sequentially to eligible population assigned to each one of the Basic Healthcare Areas.

Subjects who did not meet the inclusion criteria for CRC screening were excluded according to the following criteria: personal history of CRC or adenomas, familial CRC cancer, inflammatory bowel disease, colonoscopy in the previous 5 years or faecal occult blood test (FOBT) in the 
previous 2 years, terminal disease or severe disabling condition. Subjects with an invalid they could not be invited to screening.

Eligible subjects were invited by a personal letter offering a test completely free, a leaflet with information regarding $\mathrm{CRC}$ and the screening process and a list of the nearest pharmacies participating in the program. A reminder letter was sent out if no response was obtained after six weeks of the initial invitation.

A detailed description of the FIT performance is provided elsewhere (Garcia et al. 2015). Briefly, participants collected one sample of feces from one bowel movement and a $100 \mathrm{ng} \mathrm{Hb} / \mathrm{ml}$ cutoff (20 mg Hb/g feces) was used as the threshold for test positivity. Those individuals with a positive test were offered a colonoscopy for diagnostic confirmation.

\section{$\underline{\text { Randomized field trial }}$}

We conducted a four-arm randomized field trial to investigate the efficacy of the implementation of an intention prompt and the additional messages.

\section{Invitation mailing conditions}

As behavioral interventions are most likely to influence screening if they target more than one factor, we combined the implementation prompt in two experimental conditions with either the message about the high prevalence or importance of regular screening (Power et al. 2009; Camiloni et al. 2013; Senore et al. 2015).

The control group was the standard invitation letter used in the other screening areas In addition, the three intervention conditions prompted recipients to write down the date they planned to pick up their test kit and two of them included additional information about injunctive social norms or benefit of early detection. A standard reminder letter was sent out if no response was obtained after six weeks of the initial invitation.

The intention prompt on top of the sheet with the engaged pharmacies was adapted from a previous study and contained the sentence: "The majority people find it helpful to write down the date they want to pick up their test kit" (Milkman et al. 2011). The normative message on the social norms condition stated the proportion of individuals who engage in the desired behavior (Jemmot et al. 1986). The sentence said: "The majority of people participating for the first time continue in the program later on. Follow their example and participate now." The benefit 
condition added the following sentence to the invitation letters: "Regular participation in the screening program increases the chance of early detection and facilitates treatment." The reminder letter was sent out six weeks after the initiation letter and was identical for all experimental conditions.

\section{Study population}

Our study population was 5,077 inhabitants (from a basic healthcare area) eligible for the sixth biennial CRC screening program of L'Hospitalet of Llobregat. These subjects were invited from the $12^{\text {th }}$ of May to the $4^{\text {th }}$ of July 2014 ,

A quarter of the population was assigned to each experimental condition $(n=4)$. Intervention and control groups were randomized along the 8 week-period to prevent summer break effects. Figure 1 shows the component of the mailer that varied across the four conditions and the distribution of invitations throughout the period of study. There were no differences in the distribution of individuals between the four mailing conditions in terms of sex $(p=0.78)$, age $(p=0.64)$ and CRC screening experience $(p=1.00)$.

The study protocol was approved by the Clinical Research Ethics Committee of the Bellvitge University Hospital, and all involved parties followed the ethical requirements set forth in the Spanish Organic Law on Protection of Personal Data (15/1999 of December 13).

The outcomes of interest were participation rates according to each experimental condition before and after the reminder letter.

We performed a logistic regression with random intercept analysis to compare each mailing condition versus the standard invitation letter, adjusting for sex and age group. We included the invitation week as random effects intercept to account for the cluster variability. We stratified the model by previous screening behavior. We reported the odds ratio (OR) adjusted for age and sex and $95 \%$ confidence intervals $(95 \% \mathrm{Cl})$. This study had $80 \%$ power to detect differences in participation of $5.5 \%$ between control and experimental groups.

\section{Results}


The overall participation in the CRC screening program was 34.6\%. From those individuals who picked-up the FIT at the pharmacies, $96.8 \%$ completed and returned it (data not shown).

Table 1 shows the effect of reminder letter on participation rates according to previous screening behavior. An overall 8.0 percentage point increase in CRC screening was achieved, which translates to an additional 409 individuals who were screened, as a direct result of receiving a reminder. Differential rates were statistically significant in all groups (newly invited, never screened and previous screened).

Table 2 presents participation rates according to the different behavioral interventions. Overall, participation rates among those individuals allocated in the prompt and the benefit conditions were lower than those in the control and norms condition.

Multivariate logistic regression analysis showed that the odds for completing a screening test were significantly lower for two subgroups (table 3). Newly invited individuals were less likely to hand in their stool samples if they were in the benefit condition (OR: 0.60; 95\% Cl: 0.38-0.95). On the other hand, individuals who had been previously screened might be less likely to participate when they were allocated in the prompt condition compared to those who got the standard invitation letter. Although after adjusting for the cluster effect the differences were not statistically significant (OR: $0.68 ; 95 \% \mathrm{Cl}: 0.40-1.17)$.

\section{Discussion}

While none of our three experimental conditions increased screening behavior, we found that highlighting the benefit of continuous behavior may discourage individuals to adapt. The results of this study demonstrate a clear demotivating effect of praising the benefits of regular participation on individuals who had never been invited before. In designing invitation mailings, one should be very cautious in stating the desired behavior, because repeated behavior may be perceived as a large request. Studies have shown that individuals are more likely to agree to a small than a large request (Freedman and Fraser, 1996; Dolin and Booth-Butterfield, 1995). Essentially, the more an individual goes along with small requests, like participating in a screening program one time, the more likely is it that the individual participates also in future programs. In this sense, the newly invited individual may have felt obligated to participate regularly. 
The insignificant impact of the implementation prompt suggests that individuals do not have finding of the implementation prompt in the context of vaccination. It may be that getting a flu shot is more time consuming and therefore more difficult to plan than passing by a pharmacy to pick up the test kit. This argument is supported by the finding of the original study that the implementation prompt had a larger impact at one-day clinic sites than at multi-day clinic sites (Milkman et al, 2011). The authors believe that the implementation intentions prompts are most effective when the opportunity for participation is fleeting. In our case, the pharmacies are at least as easily accessible as multi-day clinics and the implementation prompt may therefore not be needed.

Differently, the insignificant effect of the social norms treatment may have been caused by the vague description of the participation rate. Stating that the majority of individuals participating for the first time continue in the program later on does not give individuals a clear picture of the social norm. It may be that individuals think that only slightly more than $50 \%$ of the individuals continue with the program. A future study should therefore investigate whether communicating the observed $80 \%$ participation rate of individuals who have participated previously affects screening behavior.

Finally, we found that the neutral reminder letter increased screening rates, independently from the individual's experience with the screening program $(p<0.01)$. This positive effect of the reminder letter is persistent with previous research (Senore et al. 2015; Brouwers et al. 2011a; Brouwers et al. 2011b; Sabatino et al. 2012).

A systematic review study showed that an advance notification letter, postal mailing, written reminders increase uptake of faecal tests for CRC screening uptake and could be easily implemented (Rat el al., 2018). Some studies assessed the effect of different frames of invitation messages (varying presentation and content of the written information). They showed increases in uptake, on the basis of a leaflet containing information on FOBT or an ‘implementation intention' technique (Rat et al., 2018).

Previous research supports that the low prevalence of screening for colorectal cancer appears to be due to lack of awareness and inadequate provider counseling rather than poor patient acceptance for screening. Systematic counseling about colorectal cancer screening will likely 
improve screening rates and reduce disparities by race/ethnicity and education (Gilbert and Kanarek, 2005; Wee, McCarthy and Phillips, 2005). Nevertheless, communication interventions to improve CRC screening rates must therefore be appropriate in terms of cognitive and health literacy demands (Smith et al., 2015). Feedback between the CRC screening programme and primary health care professionals is essential to ensure the quality of the screening programme and consistent monitoring criteria (Benito et al., 2016)

This study has some limitations, we could not randomize at individual level because of the CRC screening invitation process. CRC screening territory is divided according to healthcare basic areas, and individuals are invited sequentially. As cluster randomization is more prone to bias than individual randomization we included the invitation week as a random effect in the model to account for that variability.

Although the impacts of our experimental conditions were mostly insignificant, our study shows that importance of proof-of-concept experiments to generate reliable evidence of the interventions. Implementation prompts have been used successfully in the context of vaccination (Milkman et al. 2011), but they did not work in a setting where participation was less time consuming. It is therefore important to provide reliable evidence of the impact of behavioral interventions for each application. A basic requirement for scientific integrity is the ability to replicate the results of research and apply them to different settings. These proof-of-concept studies are particularly important to generalize findings.

\section{Acknowledgments}

This study was partially co-funded by the Carlos III Health Institute and the European Regional Development Fund - a way to build Europe (PI05/0942, RD12/003670053), and by the Department of Universities and Research (2014SGR635, 2014SGR647), Government of Catalonia.

\section{Declaration of conflicting interests}

The authors declared no potential conflict of interest with respect to the research, authorship and /or publication of this article. 


\section{References}

Baker, D. W., Brown, T., Buchanan, et al. (2014). Comparative effectiveness of a multifaceted intervention to improve adherence to annual colorectal cancer screening in community health centers: a randomized clinical trial. JAMA internal medicine, 174(8), 1235-1241.

Benito, L., García, M., Binefa, G., et al. (2016). Cross-sectional survey on awareness of colorectal cancer and a screening programme for primary health care professionals in Catalonia, Spain. European journal of cancer care, 25(6), 992-1004.

Brouwers MC, De Vito C, Bahirathan L, et al (2011) Effective interventions to facilitate the uptake of breast, cervical and colorectal cancer screening: an implementation guideline. Implement Sci;6(1):112.

Brouwers MC, De Vito C, Bahirathan L, et al (2011) What implementation interventions increase cancer screening rates? a systematic review. Implement Science; 6 (1): 111.

Bulliard JL, Garcia M, Blom J, Senore C, Mai V, Klabunde C. Sorting out measures and definitions of screening participation to improve comparability: The example of colorectal cancer. Eur J Cancer 2014;50(2): 434-46.

Camilloni L, Ferroni E, Cendales BJ, et al (2013) Methods to increase participation in organized screening programs: a systematic review. BMC Public Health;13 (1): 464.

Cialdini RB, Reno RR, Kallgren CA (1990) A focus theory of normative conduct: recycling the concept of norms to reduce littering in public places. J Pers Soc Psychol; 58(6):1015-26.

Dolin DJ, Booth-Butterfield S (1995) Foot-in-the-door and cancer prevention. Health Commun;7(1):55-66.

Freedman JL, Fraser SC (1996) Compliance without pressure: the foot-in-the-door technique. J Pers Soc Psychol;4(2):195-202.

Garcia M, Borràs JM, Milà N, et al (2011) Factors associated with initial participation in a population-based screening for colorectal cancer in Catalonia, Spain: a mixed-methods study. Prev Med;52 (3):265-7

Garcia M, Milà N, Binefa G et al (2015) Fecal hemoglobin concentration as a measure of risk to tailor colorectal cancer screening: are we there yet? Eur J Cancer Prev;24(4):321-27.

Gilbert A, Kanarek N. Colorectal cancer screening: physician recommendation is influential advice to Marylanders. Prev Med. 2005;41(2):367-79. doi:10.1016/j.ypmed.2005.01.008.

Gollwitzer PM (1999) Implementation intentions: strong effects of simple plans. Am Psychol; 54 (7): 493-503.

Gollwitzer PM, Sheeran P (2006) Implementation intentions and goal achievement: A meta-analysis of effects and processes. Adv Exp Soc Psychol; 38: 69-119.

Hassan C, Giorgi Rossi P, Camilloni L, et al (2012) Meta-analysis: adherence to colorectal cancer screening and the detection rate for advanced neoplasia, according to the type of screening test. Aliment Pharmacol Ther; 36 (10):929-40.

Javanparast, S., Ward, P., Young, G et al. (2010). How equitable are colorectal cancer screening programs which include FOBTs? A review of qualitative and quantitative studies. Preventive medicine, 50(4), 165-172. 
Jemmott JB , Ditto PH, Coryle RT (1986). Judging health status: effects of perceived prevalence and personal relevance. J Pers Soc Psychol;50(5):899-905.

Karsa, L. V., Anttila, A., Ronco, G., Ponti, A., Malila, N., Arbyn, M., ... \& Hery, C. (2008). Cancer screening in the European Union. Report on the implementation of the Council Recommendation on cancer screening. Cancer screening in the European Union. Report on the implementation of the Council Recommendation on cancer screening.

Klabunde C, Blom J, Bulliard JL, et al. (2015) Participation rates for organized colorectal cancer screening programmes: an international comparison. J Med Screen; 22 (3):119-26.

Milkman KL, Beshears J, Choi JJ, et al (2011) Using implementation intentions prompts to enhance influenza vaccination rates. Proc Natl Acad Sci USA.; 108 (26): 10415-20.

Molina-Barceló, A., Salas Trejo, D., Peiró-Pérez, R., \& Málaga López, A. (2011). To participate or not? Giving voice to gender and socio-economic differences in colorectal cancer screening programmes. European journal of cancer care, 20(5), 669-678.

Power E, Miles A, von Wagner C, et al (2009) Uptake of colorectal cancer screening: system, provider and individual factors and strategies to improve participation. Future Oncol; 5(9):137188.

Purnell JQ, Thompson T, Kreuter MW, McBride TD. Behavioral Economics: "Nudging" Underserved Populations to Be Screened for Cancer. Prev Chronic Dis 2015;12:140346. DOI: http://dx.doi.org/10.5888/ pcd12.140346

Rat, C., Latour, C., Rousseau, R., Gaultier, A., Pogu, C., Edwards, A., \& Nguyen, J. M. (2018). Interventions to increase uptake of faecal tests for colorectal cancer screening: a systematic review. European Journal of Cancer Prevention, 27(3), 227-236.

Sabatino SA, Lawrence B, Elder R, et al (2012). Community Preventive Services Task Force. Effectiveness of interventions to increase screening for breast, cervical, and colorectal cancers: nine updated systematic reviews for the guide to community preventive services. Am J Prev Med;43(1):97-118.

Senore C, Inadomi J, Segnan N, Bellisario C, et al (2015) Optimising colorectal cancer screening acceptance: a review. Gut; 64 (7):1158-77.

Smith SG, Vart G, Wolf MS, Obichere A, Baker HJ, Raine R, Wardle J, von Wagner C. How do people interpret information about colorectal cancer screening: observations from a think-aloud study. Health Expect. 2015 Oct;18(5):703-14. doi: 10.1111/hex.12117.

Solar O, Irwin A. A conceptual framework for action on the social determinants of health. Social Determinants of Health Discussion. Paper 2 (Policy and Practice).

Wee CC, McCarthy EP, Phillips RS. Factors associated with colon cancer screening: the role of patient factors and physician counseling. Prev Med. 2005;41(1):23-9.

Weller DP, Patnick J, McIntosh HM, et al (2009) Uptake in cancer screening programmes. The Lancet Oncol; 10 (7):693-9. 
Figure 1: Component of the invitation mailers

1

2

3

4

5

7

8

10

11

12

13

14

15

16

17

18

19

20

21

22

23

24

25

26

27

28

29

30

31

32

33

34

35

36

37

38

39

40

41

42

43

44

45

46

47

48

49

50

51

52

53

54

55

56

57

58

59

60

61

62

63

64

65 
Figure 1: Component of the invitation mailers

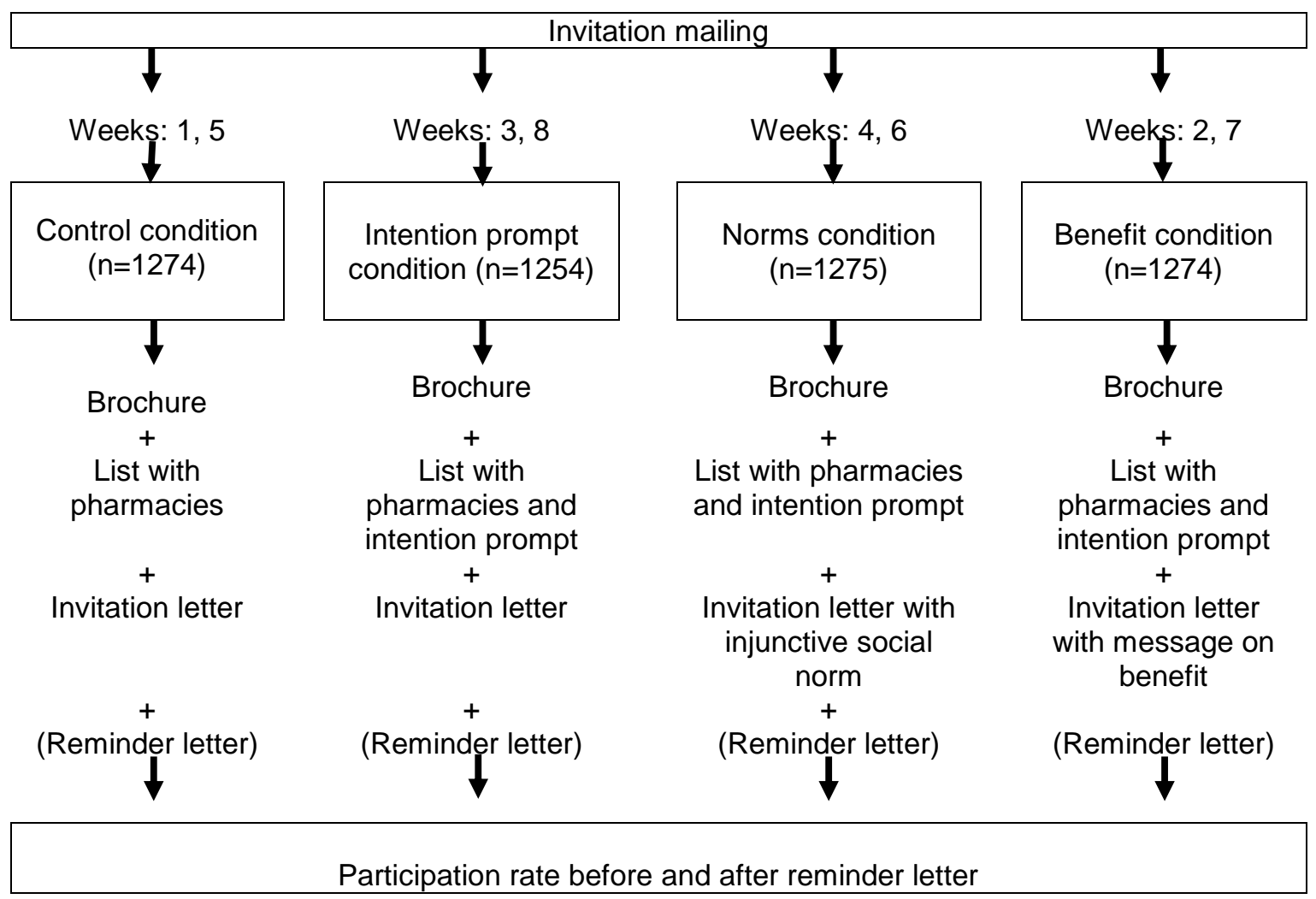


Table 1. CRC screening uptake before and after the reminder letter.

\begin{tabular}{|c|c|c|c|c|}
\hline & \multicolumn{2}{|c|}{$\begin{array}{l}\text { Initial participation } \\
\text { (before reminder) }\end{array}$} & \multicolumn{2}{|c|}{$\begin{array}{c}\text { Final participation } \\
\text { (after reminder) }\end{array}$} \\
\hline & $\%$ & $95 \% \mathrm{Cl}$ & $\%$ & $95 \% \mathrm{Cl}$ \\
\hline Never screened $(n=2,856)$ & 7.1 & $6.22-8.11$ & 11.4 & $10.3-12.6$ \\
\hline Newly invited ( $\mathrm{n}=738)$ & 18.6 & $15.9-21.5$ & 30.8 & $27.5-34.2$ \\
\hline Previously screened $(n=1,483)$ & 67.4 & $64.9-69.7$ & 80.6 & $78.5-82.5$ \\
\hline Overall $(n=5,077)$ & 26.4 & $25.2-27.6$ & 34.4 & $33.1-35.7$ \\
\hline
\end{tabular}

Table 2. Participation rates among individuals invited for CRC screening according to behavioural interventions.

Final participation

\begin{tabular}{lccc} 
& No & Yes & $\%$ \\
\cline { 2 - 4 } All participants $(n=5,077)$ & & & \\
$\quad$ Control & 817 & 457 & 35.9 \\
Prompt & 850 & 404 & 32.2 \\
$\quad$ Social Norms & 816 & 458 & 35.9 \\
$\quad$ Benefit & 846 & 429 & 33.6 \\
Newly invited $(n=738)$ & & & \\
$\quad$ Control & 121 & 65 & 34.9 \\
Prompt & 128 & 52 & 28.9 \\
$\quad$ Social Norms & 121 & 65 & 34.9 \\
$\quad$ Benefit & 141 & 45 & 24.2 \\
Never screened $(n=2,856)$ & & & \\
$\quad$ Control & 632 & 84 & 11.7 \\
$\quad$ Prompt & 636 & 73 & 10.3 \\
$\quad$ Social Norms & 623 & 93 & 13.0 \\
$\quad$ Benefit & 639 & 76 & 10.6 \\
Previously screened $(n=1,483)$ & & & \\
$\quad$ Control & 64 & 308 & 82.8 \\
$\quad$ Prompt & 86 & 279 & 76.4 \\
$\quad$ Social Norms & 72 & 300 & 80.6 \\
$\quad$ Benefit & 66 & 308 & 82.4 \\
\hline
\end{tabular}


Table 3. Multivariate logistic regression models to evaluate CRC screening uptake according to three behavioral interventions and stratified by previous screening behavior.

\begin{tabular}{|c|c|c|c|c|c|c|c|c|}
\hline & \multicolumn{2}{|c|}{ Overall } & \multicolumn{2}{|c|}{ Newly invited } & \multicolumn{2}{|c|}{ Never screened } & \multicolumn{2}{|c|}{ Previously screened } \\
\hline & OR & $(95 \% \mathrm{Cl})$ & OR & $(95 \% \mathrm{Cl})$ & OR & $(95 \% \mathrm{Cl})$ & OR & $(95 \% \mathrm{Cl})$ \\
\hline Control & 1 & & 1 & & 1 & & 1 & \\
\hline Prompt & 0.85 & $(0.75-1.08)$ & 0.76 & $(0.49-1.18)$ & 0.86 & $(0.62-1.20)$ & 0.68 & $(0.40-1.17)$ \\
\hline Social Norms & 1.00 & $(0.83-1.20)$ & 1.00 & $(0.65-1.53)$ & 1.12 & $(0.82-1.54)$ & 0.86 & $(0.50-1.47)$ \\
\hline Benefit & 0.90 & $(0.75-1.08)$ & 0.60 & $(0.38-0.95)$ & 0.89 & $(0.64-1.23)$ & 0.95 & $(0.55-1.64)$ \\
\hline
\end{tabular}

Models adjusted for sex and age 
Conflict of Interest Form
Click here to download C

Click here to download Conflict of Interest Form: 2016 coi_ypmed.doc 
LaTeX Source Files
Click here to download LaTeX Source Files: Title Page.docx

LaTeX Source Files
Click here to download LaTeX Source Files: Title Page.docx Click here to download LaTex Source Files: Title Page.docx

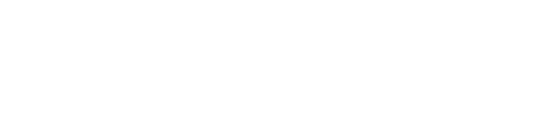

$\sqrt{2}$

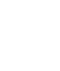

(1)

(1)

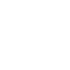
(1) (1) (1) (1) (1) . . . . . . . .

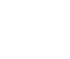

